\title{
Low-dose-intensity bevacizumab with weekly irinotecan for platinum- and taxanes-resistant epithelial ovarian cancer
}

\author{
Ying Liu $\cdot$ Zhonghai Ren $\cdot$ Shuning Xu $\cdot$ Hua Bai $\cdot$ \\ Ning Ma $\cdot$ Feng Wang
}

Received: 29 October 2014 / Accepted: 8 January 2015 / Published online: 20 January 2015

(C) The Author(s) 2015. This article is published with open access at Springerlink.com

\begin{abstract}
Purpose The purpose of this study was to evaluate the safety and efficacy of low-dose-intensity bevacizumab and weekly irinotecan as salvage treatment for patients with platinum- and taxanes-resistant advanced epithelial ovarian cancer.

Methods Fifty-two patients with platinum- and taxanesresistant advanced epithelial ovarian cancer received bevacizumab $5 \mathrm{mg} / \mathrm{Kg}$ days 1 and 15 ; irinotecan $60 \mathrm{mg} / \mathrm{m}^{2}$ days 1,8 and 15 . The combined therapy was repeated every 28 days, up to six cycles.

Results A total of 230 cycles of bevacizumab combined with irinotecan were administrated to 52 patients. Among the 52 patients, 22 patients achieved partial response $(42.3 \%) ; 12$ patients had stable disease $(23.1 \%)$ and 18
\end{abstract}

Ying Liu and Zhonghai Ren have contributed equally to this work.

\section{Y. Liu $(\bowtie) \cdot$ S. Xu}

Department of Oncology, Henan Cancer Hospital, Zhengzhou

University Affiliated Cancer Hospital, Zhengzhou, Henan, China

e-mail: yaya7207@126.com

\section{Z. Ren}

Department of Oncology, Nanyang City Center Hospital,

Nanyang, Henan, China

H. Bai

Department of Oncology, People's Hospital of Zhengzhou,

Zhengzhou, Henan, China

\section{N. Ma}

Department of Oncology, Henan Provincial People's Hospital,

Zhengzhou, Henan, China

F. Wang

Department of Oncology, The First Affiliated Hospital

of Zhengzhou University, Zhengzhou, Henan, China patients experienced disease progression $(34.6 \%)$. The median progression-free survival and the median overall survival were 8.0 months $(95 \%$ confidence interval: 6.74-9.26 months) and 13.8 months (95\% confidence interval: $11.97-15.63$ months), respectively. The most frequent grade 3-4 hematologic toxicities were neutropenia $(11.5 \%)$ and thrombocytopenia $(3.8 \%)$. The non-hematologic toxicities included grade 3 diarrhea $(3.8 \%)$ and hypertension $(3.8 \%)$. Two patients $(3.8 \%)$ were confirmed with deep vein thrombosis. Febrile neutropenia, symptomatic cardiac dysfunction and gastrointestinal perforation were not observed in this study.

Conclusions The combination of low-dose-intensity bevacizumab and weekly irinotecan was an effective and safe regimen for patients with platinum- and taxanes-resistant epithelial ovarian cancer.

Keywords Bevacizumab - Irinotecan ·

Platinum-/taxanes-resistant $\cdot$ Ovarian cancer

\section{Introduction}

Ovarian cancer is the leading cause of death in women with gynecological cancer. Each year, over 2,25,000 cases are diagnosed, and about 1,40,200 women die of the disease worldwide [1]. More than $70 \%$ of patients are diagnosed with advanced ovarian cancer, and the prognoses for these patients are very poor. The 5-year survival rate for stage III cases is $23-41 \%$, and it is only $11 \%$ for stage IV cases [2]. Currently, surgical tumor debulking followed by platinum- and taxanes-based chemotherapy is the standard therapy for advanced ovarian cancer [3]. The objective response rate (ORR) of the standard therapy for ovarian cancer exceeds $80 \%$. However, $70 \%$ of patients 
who have received cytoreduction and chemotherapy will experience recurrence within 5 years due to development of resistance to platinum and taxanes [4]. Patients who suffer disease relapse within 6 months after platinum- or taxanescontaining therapy are considered to be platinum or taxanes resistant. Previous studies have shown that combination chemotherapy increased drug-related toxicity and has no survival benefit to patients with platinum- and taxanesresistant advanced epithelial ovarian cancer [5, 6]. Nowadays, the standard salvage treatment for these patients is monotherapy without platinum and taxanes. For instance, topotecan [7], liposomal doxorubicin [8], gemcitabine [9] and vinorelbine [10] have all been utilized in practice. The ORR of these single chemotherapy regimens ranges from 10 to $30 \%$ without survival benefit. Therefore, novel treatment strategies are needed to improve the overall survival (OS) of patients with platinum- and taxanes-resistant advanced ovarian cancer.

In addition to traditional cytotoxic chemotherapeutics, molecular targeted drugs have emerged as important weapons in the arsenal of cancer treatment. Anti-angiogenesis prevents the growth of blood vessels that feed tumors and has been proven to be one of the most vital pathways for targeted cancer therapies. In ovarian cancer, over expression of vascular endothelial growth factor (VEGF) has been observed and several studies have verified that high level of VEGF not only increases the risk of ascites but is also closely related to the poor prognosis [11-13]. Therefore, angiogenesis is thought to be a promising target for treating ovarian cancer. Bevacizumab is a recombinant humanized monoclonal IgG1 antibody which targets vascular endothelial growth factor (VEGF)-A. IgG1 antibody is able to inhibit tumor growth and metastasis by binding to and neutralizing all biologically active forms of VEGF-A [14]. Furthermore, anti-VEGF drugs such as bevacizumab are thought to enhance the effect of chemotherapeutic agents by normalization of tumor vessels, leading to increased tumor oxygenation and better delivery of cytotoxic drugs [15].

Bevacizumab (either alone or combined with chemotherapy) has been shown to be effective for patients with recurrent ovarian cancer. Two early phase II studies of bevacizumab monotherapy at a dose of $15 \mathrm{mg} / \mathrm{Kg} / 3$ weeks for patients with relapsing ovarian cancer achieved an ORR of $15.9-21 \%$ [16, 17]. In addition, several other phase II studies evaluated the safety and activity of bevacizumab of $10 \mathrm{mg} / \mathrm{Kg} / 2$ weeks or $15 \mathrm{mg} / \mathrm{Kg} / 3$ weeks in combination with different cytotoxic agents in advanced ovarian cancer [18-20]. These results demonstrated that highdose-intensity ( $5 \mathrm{mg} / \mathrm{Kg} /$ week) bevacizumab combined with chemotherapy improved the ORR and prolonged the progression-free survival (PFS) of patients with platinum- and taxanes-resistant ovarian cancer. However, these high-dose-intensity bevacizumab ( $5 \mathrm{mg} / \mathrm{Kg} /$ week) combination regimens resulted in more severe adverse effects than the single agents, especially increasing the incidence of bevacizumab-related gastrointestinal perforation and cardiovascular toxicities [21]. Encouragingly, a 2014 phase III study investigating similar doses of bevacizumab combined with chemotherapy for platinum-resistant ovarian cancer has reported a low GI perforation (non-fatal and fatal) of $2.2 \%$ in the AURELIA study [22].

In this study, we aim to develop a safer yet still effective bevacizumab-based combination therapy. Thus, we reasoned that lowering the dose of bevacizumab in conjunction with a carefully chosen chemotherapy might achieve a balance between efficacy and toxicity. In fact, beneficial effects of lower dose-intensity of bevacizumab $(2.5 \mathrm{mg} / \mathrm{Kg} /$ week $)$ have been shown in a recent single-agent study in relapsing ovarian cancer [23]. In another phase III trial ICON7, the dose of $7.5 \mathrm{mg} / \mathrm{Kg} / 3$ weeks as first-line treatment in ovarian cancer was shown to be much safer with risk of $1 \%$ GI perforation [24]. In terms of selection of chemotherapy agent, we decided to choose irinotecan, which has demonstrated positive effect in numerous cancer therapies. The ORR of single irinotecan for platinum- and taxanes-resistant ovarian cancer is $29 \%$ with good tolerance [25]. In addition, different dosing schedules have been developed for irinotecan, and the weekly administration regimen showed moderate efficacy with fewer cases of myelosuppression and diarrhea [26]. The proven clinical activities of bevacizumab and irinotecan as monotherapy, the potentially synergistic anti-angiogenic effects and their non-overlapping toxicity profiles all provide compelling rationale for combining the two drugs for patients with advanced ovarian cancer. Currently, no study of bevacizumab combined with irinotecan for advanced ovarian cancer has been documented. Therefore, we designed this study to evaluate the safety and efficacy of low-dose-intensity bevacizumab and weekly irinotecan as salvage chemotherapy for patients with platinum- and taxanes-resistant advanced ovarian cancer.

\section{Methods}

\section{Study design}

This study was a phase II trial and was designed to evaluate the efficacy and safety of six cycles of bevacizumab plus irinotecan in platinum- and taxanes-resistant advanced ovarian cancer patients. This study was initiated in November 2010 and completed enrollment in May 2013. Local ethics committee approval was obtained before any patient was enrolled into the study which was performed in accordance with the Declaration of Helsinki. All patients signed informed consent before study entry. 
Patients' eligibility

Eligibility criteria for study entry were as follows: patients were at least 18 years of age with cytologically or histologically proved epithelial ovarian or fallopian tube cancer. All patients received prior primary platinum- and taxanes-based first-line chemotherapy. Patients required measurable disease according to response evaluation criteria in solid tumors (RECIST) [27], Eastern Cooperative Oncology Group (ECOG) performance status $\leq 2$, adequate bone marrow (absolute neutrophil count $\geq 1.5 \times 10^{9} / \mathrm{L}$, platelet count $\geq 100 \times 10^{9} / \mathrm{L}$, hemoglobin $\geq 90 \mathrm{~g} / \mathrm{L}$ ), normal hepatic function (bilirubin $\leq 1.5 \times$ the upper limit of normal, alanine aminotransferase and aspartate aminotransferase $\leq 2.5 \times$ the upper limit of normal), normal renal function(serum creatinine $\leq 1.5 \times$ the upper limit of normal or creatinine clearance $>60 \mathrm{ml} /$ min), normal cardiac function. Furthermore, patients had life expectancy $\geq 12$ weeks. All patients signed informed consent before treatment.

Exclusion criteria included a concurrent malignancy; significant medical comorbidities; clinically significant cardiovascular disease including uncontrolled hypertension, myocardial infarction and unstable angina; New York Heart Association grade 2 or greater congestive heart failure; history of active bleeding; major surgery within 28 days before the initiation of study treatment; serious infections, non-healing wound, ulcer or bone fracture; pregnancy or lactation; known central nervous system metastases; bowel obstruction; and inability to sign informed consent. Patients who have been exposed to topotecan, irinotecan or bevacizumab were excluded.

\section{Treatment and dose modification}

Eligible patients received bevacizumab (Avastin: Roche, Basel, Switzerland) $5 \mathrm{mg} / \mathrm{Kg}$ days 1 and 15 ; irinotecan (Campto: Pfizer, New York, NY, U.S.A.) $60 \mathrm{mg} / \mathrm{m}^{2}$ days 1 , 8 and 15 . The dose cycles were repeated every 28 days for a maximum of six cycles. Bevacizumab was given by intravenous over $90 \mathrm{~min}$ for the first time and $30 \mathrm{~min}$ for subsequent infusion. Irinotecan was administered intravenously over 60-90 min.

There was no dose modification of bevacizumab. According to the adverse events, bevacizumab was either given or withheld. If the patients experienced severe bevacizumab-related toxicities such as symptomatic cardiac dysfunction, uncontrolled hypertension grade 4 , hemorrhage grade 3-4, grade 4 proteinuria, any grade of arterial thrombosis and gastrointestinal perforation, they were removed from the study. Dose of irinotecan was reduced sequentially to $50 \mathrm{mg} / \mathrm{m}^{2}$ and to $40 \mathrm{mg} / \mathrm{m}^{2}$ for grade 3-4 toxicities or for a treatment-related toxicity causing delay in treatment. Irinotecan was omitted if patients had hematologic toxicity of grade 4 or non-hematologic toxicity grade $\geq 3$ on the day of the schedule administration. If patients experienced toxicity grade $\geq 3$ after two dose reduction of irinotecan, they were withdrawn from the study. Treatment did not stop until one of the following criteria was met: disease progression, unacceptable toxicity, patient refusal, or need to delay chemotherapy more than 3 weeks.

\section{Response and toxicity assessment}

Patients were considered assessable if they had completed at least two cycles of chemotherapy or were removed from study due to disease progression. Baseline evaluation consisted of a complete history and physical examination that included a gynecological examination, laboratory studies including CA-125 marker analysis and dynamic contrastenhanced computed tomography (CT) or magnetic resonance imaging (MRI) scans within 4 weeks of study entry. Tumor objective response was evaluated every two cycles during therapy and every 8 weeks during follow-up according to RESIST [27] by dynamic contrast-enhanced CT or MRI scans, including the confirmation of response. The level of CA-125 change was not used as the standard for response evaluation. All patients who received at least one dose of study treatment were evaluated for toxicity. Toxicities were assessed and graded according to common terminology criteria for adverse events (NCI-CTCAE) version 4.0 [28] every dose cycle.

\section{Statistical analysis}

The primary endpoints of this phase II study were safety and PFS; the secondary endpoints were OS and ORR. Based on the recent studies of platinum- and taxanesresistant recurrent epithelial ovarian cancer, the null hypothesis set a PFS of 4.5 months for non-platinum chemotherapy without bevacizumab. The alternative hypothesis proposed a PFS of 7.5 months. A two-sided log-rank test with a sample size of 46 assessable patients achieves $80 \%$ power at $5 \%$ type I error level to reject the null hypothesis. Progression-free survival was measured from the date of enrollment to the date of the first observation of disease progression or the date of death for any cause if there had been no progression. Patients without disease progression at the time of analysis or at time of death were censored at their last date of efficacy evaluation. Overall survival was measured from the date of enrollment until the date of death for any cause. Overall survival was censored at the last date of follow-up. PFS and OS curves were calculated using the KaplanMeier method. 


\section{Results}

Patient characteristics

From November 2010 to May 2013, a total of 52 patients were recruited. All the patients were evaluated for toxicity and efficacy. Demographics are listed in Table 1. The median age was 55 years (range 33-65 years), and the median ECOG performance status was 0 (range 0-2). Forty-two of 52 patients $(80.8 \%)$ experienced surgical tumor debulking. The median number of prior chemotherapy regimen was 2 (range $1-3$ ).

\section{Clinical efficacy}

Fifty-two eligible patients received 230 cycles of bevacizumab plus irinotecan treatment in all. None of the patients achieved complete response, 22 patients experienced partial response $(42.3 \%), 12$ patients had stable disease $(23.1 \%)$, and the other 18 patients were confirmed with progressive disease $(34.6 \%)$. The ORR was $42.3 \%$, and disease control rate was up to $65.4 \%$ (Table 2). A total of 48 deaths were observed among the 52 patients during or after the therapy. The median PFS (Fig. 1) and median OS (Fig. 2) were 8.0 months (95\% confidence interval: $6.74-$ 9.26 months) and 13.8 months (95\% confidence interval: 11.97-15.63 months), respectively.

\section{Toxicity}

All of the 52 patients who received the bevacizumab plus irinotecan therapy were assessable for safety analysis. There was no therapy-related death. Table 3 lists the most common adverse events. The grade 3 hematologic toxicities were neutropenia $(11.5 \%)$ and thrombocytopenia $(3.8 \%)$. Grade 4 hematologic toxicity was not observed in our study. Among the 52 patients, two patients (3.8\%) experienced grade 3 diarrhea with cramp. two patients $(3.8 \%)$ received grade 3 hypertension, and two patients $(3.8 \%)$ was confirmed with deep vein thrombosis after four cycles of combination therapy. There was no febrile neutropenia, symptomatic cardiac dysfunction, grade 4 hypertension, hemorrhage or gastrointestinal perforation.

\section{Discussion}

As suggested by our data presented above, the combination of low-dose-intensity bevacizumab ( $5 \mathrm{mg} / \mathrm{Kg} / 2$ weeks) and weekly irinotecan $\left(60 \mathrm{mg} / \mathrm{m}^{2} \mathrm{~d} 1,8,15 \mathrm{q} 28 \mathrm{~d}\right)$ seems to be an effective treatment with moderate toxicity in patients with platinum- and taxanes-resistant advanced epithelial ovarian cancer.
Table 1 Patient demographics

\begin{tabular}{lc}
\hline Demographics & $\begin{array}{l}\text { Bevacizumab and irinotecan } \\
N=52\end{array}$ \\
\hline Median age (years) & $55(33-65)$ \\
ECOG performance status & $36(69.2)$ \\
0 & $12(23.1)$ \\
1 & $4(7.7)$ \\
2 & \\
Primary site, no (\%) & $8(15.4)$ \\
Fallopian tube & $44(84.6)$ \\
Ovarian & \\
Histology, no (\%) & $38(73.1)$ \\
Serous & $8(15.4)$ \\
Endometrioid & $6(11.5)$ \\
Mixed pattern & \\
Disease stage, no (\%) & $12(23.1)$ \\
II & $26(50.0)$ \\
III & $14(26.9)$ \\
IV & \\
Cytoreductive surgery, no (\%) & $42(80.8)$ \\
Yes & $10(19.2)$ \\
No & \\
Prior regimen, no (\%) & $14(27.0)$ \\
1 & $32(61.5)$ \\
2 & $6(11.5)$ \\
3 &
\end{tabular}

ECOG Eastern Cooperative Oncology Group

Table 2 Objective response

\begin{tabular}{lcc}
\hline Bevacizumab and irinotecan & \\
\hline Response & $\begin{array}{l}\text { Number of } \\
\text { patients }(N=52)\end{array}$ \\
\hline Complete response & 0 & 0 \\
Partial response & 22 & 42.3 \\
Stable disease & 12 & 23.1 \\
Progressive disease & 18 & 34.6 \\
Disease control rate & 65.4 & \\
Overall response rate & 42.3 & \\
\hline
\end{tabular}

In terms of efficacy, our combination protocol displayed an ORR of $42.3 \%$ with a disease control rate of $65.4 \%$, a median PFS of 8.0 months (95\% confidence interval: 6.74-9.26 months) and a median OS of 13.8 months (95\% confidence interval: $11.97-15.63$ months). These results have shown significant improvement compared with single bevacizumab or irinotecan regimens. In a recent review of anti-angiogenic agents, single bevacizumab (15 mg/ $\mathrm{Kg} / 3$ weeks) in recurrent ovarian cancer gave an ORR of 16-21\% with a median PFS of 4.4-4.7 months [29]. As 
Fig. 1 Progression-free survival of patients with platinum- and taxanes-resistant advanced epithelial ovarian cancer received bevacizumab and irinotecan (median progression-free survival: 8.0 months [95\% confidence interval: 6.74-9.26 months])
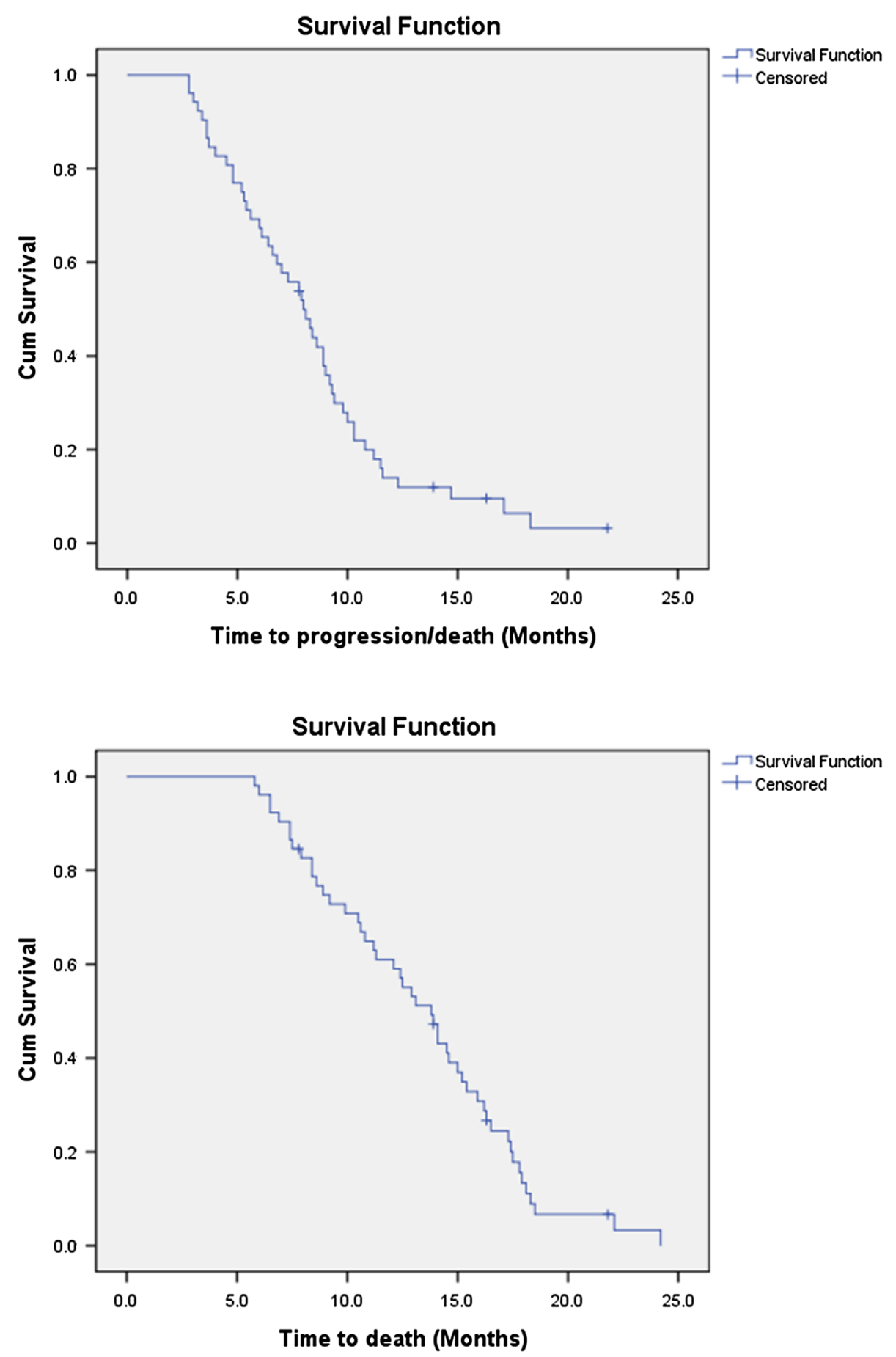

Fig. 2 Overall survival of patients with platinum- and taxanes-resistant advanced epithelial ovarian cancer received bevacizumab and irinotecan (median overall survival: 13.8 months [95 \% confidence interval: $11.97-15.63$ months]) for the single irinotecan regimen in patients with platinumresistant ovarian cancer, a phase II study demonstrated that irinotecan of $300 \mathrm{mg} / \mathrm{m}^{2} / 3$ weeks had an ORR of $17.2 \%$ and a median PFS of 2.8 months [30].

As mentioned earlier, bevacizumab has been combined with different cytotoxic agents for treatment of platinumand taxanes-resistant ovarian cancer. In all of these studies, bevacizumab was administered at higher dosage than that used in our treatment plan $(5 \mathrm{mg} / \mathrm{Kg} / 2$ weeks). For example, in a single-arm phase II study, 44 patients were treated with bevacizumab at the dose of $15 \mathrm{mg} / \mathrm{Kg} / 3$ weeks in combination with liposomal doxorubicin, yielding an ORR of $30.2 \%$ and a median PFS of 7.8 months [20], while in another phase II study, $10 \mathrm{mg} / \mathrm{Kg} / 2$ weeks of bevacizumab plus topotecan received $25 \%$ ORR with a median PFS of 7.8 months [18]. In the phase III AURELIA study, the same dose of bevacizumab plus chemotherapy in platinum-resistant ovarian cancer received $27.3 \%$ 
Table 3 Treatment-related adverse events

\begin{tabular}{|c|c|c|c|c|}
\hline \multirow[t]{2}{*}{ Adverse events } & \multicolumn{4}{|c|}{ Bevacizumab and irinotecan $(N=52)$} \\
\hline & $\begin{array}{l}\text { Grade } 1 \\
N(\%)\end{array}$ & $\begin{array}{l}\text { Grade } 2 \\
N(\%)\end{array}$ & $\begin{array}{l}\text { Grade } 3 \\
N(\%)\end{array}$ & $\begin{array}{l}\text { Grade } 4 \\
N(\%)\end{array}$ \\
\hline \multicolumn{5}{|l|}{ Hematologic } \\
\hline Neutropenia & $8(15.4)$ & $6(11.5)$ & $6(11.5)$ & 0 \\
\hline Anemia & $4(7.7)$ & $2(3.8)$ & 0 & 0 \\
\hline Thrombocytopenia & $4(7.7)$ & 0 & $2(3.8)$ & 0 \\
\hline Febrile neutropenia & 0 & 0 & 0 & 0 \\
\hline \multicolumn{5}{|l|}{ Non-hematologic } \\
\hline \multicolumn{5}{|l|}{ Gastrointestinal disorders } \\
\hline Nausea & $6(11.5)$ & $4(7.7)$ & 0 & 0 \\
\hline Vomiting & $2(3.8)$ & $2(3.8)$ & 0 & 0 \\
\hline Cramp & $2(3.8)$ & 0 & $2(3.8)$ & 0 \\
\hline Constipation & $10(19.2)$ & $2(3.8)$ & 0 & 0 \\
\hline Diarrhea & $4(7.7)$ & $2(3.8)$ & $2(3.8)$ & 0 \\
\hline Ileus & 0 & 0 & 0 & 0 \\
\hline $\begin{array}{l}\text { Gastrointestinal } \\
\text { perforation }\end{array}$ & 0 & 0 & 0 & 0 \\
\hline \multicolumn{5}{|l|}{ Neurotoxicity } \\
\hline Headache & $6(11.5)$ & 0 & 0 & 0 \\
\hline Sensory nerve disorder & $4(7.7)$ & 0 & 0 & 0 \\
\hline \multicolumn{5}{|l|}{ Renal and urinary disorders } \\
\hline Proteinuria & $2(3.8)$ & 0 & 0 & 0 \\
\hline Renal failure acute & 0 & 0 & 0 & 0 \\
\hline \multicolumn{5}{|c|}{ Cardiac and Vascular disorders } \\
\hline $\begin{array}{l}\text { Asymptomatic decrease } \\
\text { in ejection fraction }\end{array}$ & $2(3.8)$ & 0 & 0 & 0 \\
\hline $\begin{array}{l}\text { Symptomatic cardiac } \\
\text { dysfunction }\end{array}$ & 0 & 0 & 0 & 0 \\
\hline Hypertension & $6(11.5)$ & $2(3.8)$ & $2(3.8)$ & 0 \\
\hline Deep vein thrombosis & $2(3.8)$ & & & \\
\hline Arterial thrombosis & 0 & & & \\
\hline \multicolumn{5}{|l|}{ Others } \\
\hline Fatigue & $6(11.5)$ & 0 & 0 & 0 \\
\hline Appetite loss & $4(7.7)$ & $2(3.8)$ & 0 & 0 \\
\hline Alopecia & $8(15.4)$ & $4(7.7)$ & 0 & 0 \\
\hline
\end{tabular}

ORR with a median PFS of 6.7 months [22]. These results showed comparable survival benefits as those observed in our study.

Safety, as well as efficacy, is critical for patients who have received heavy chemotherapy to participate the salvage treatment. In our study, the grade 3 hematologic toxicities were neutropenia $(11.5 \%)$ and thrombocytopenia (3.8\%). Grade 3-4 non-hematologic toxicities were diarrhea $(3.8 \%)$ and hypertension $(3.8 \%)$. In addition, two patients were confirmed with deep vein thrombosis after four cycles of combination therapy. Among the 52 patients, grade 4 hematologic toxicity and bevacizumab-related adverse events such as gastrointestinal perforation, cardiac dysfunction, arterial thrombosis, grade 4 proteinuria and hypertension were not observed.

In the study of single bevacizumab, 44 patients who received $15 \mathrm{mg} / \mathrm{Kg} / 3$ weeks of bevacizumab experienced severe adverse events [17]. Bevacizumab-related grade 3-4 toxicities including hypertension $(9.1 \%)$, proteinuria $(15.9 \%)$ and bleeding $(2.3 \%)$ were observed during the therapy. Furthermore, arterial thromboembolic events occurred in three patients $(6.8 \%)$, and five patients $(11.4 \%)$ experienced gastrointestinal perforation. Eventually, high-dose-intensity bevacizumab resulted in the death of three patients [17]. The regimen of high-dose-intensity of bevacizumab ( $15 \mathrm{mg} / \mathrm{Kg} / 3$ weeks) combined with liposomal doxorubicin resulted in occurrence of more grade 3-4 adverse events including palmar and plantar erythroderma (52\%), hypertension (46\%), headache (11.4\%) and pruritus $(9.1 \%)$. As is well known, the toxicity that should raise more concern is cardiac dysfunction. In the study, seven patients (16\%) experienced an asymptomatic decrease in ejection fraction. Finally, a total of $29.5 \%$ of patients did not tolerate the $15 \mathrm{mg} / \mathrm{Kg} / 3$ weeks bevacizumab dosage and discontinued the study [20]. These studies demonstrated that high-dose-intensity of bevacizumab in combination with cytotoxic regimens displayed high risk of severe adverse events in patients with platinum-resistant advanced ovarian cancer. In our studies, low-dose-intensity of bevacizumab combined with irinotecan has greatly lowered bevacizumab-related toxicity while in the meantime shown good efficacy compared with previous phase II studies.

\section{Conclusions}

The combination of low-dose-intensity bevacizumab and weekly irinotecan provided significant efficacy with good tolerance in patients with platinum- and taxanes-resistant ovarian cancer. The results of the combination therapy warrant further investigation of a larger randomized phase III trial. Attention should be given to determine the optimal dosing schedule and to minimize treatment-related toxicities.

Acknowledgments We wish to thank Peng Liu (Ph.D.) for assistance in preparation of the manuscript.

Conflict of interest None of the authors has financial or personal relationships with other people or organizations that could inappropriately influence this work.

Open Access This article is distributed under the terms of the Creative Commons Attribution License which permits any use, distribution, and reproduction in any medium, provided the original author(s) and the source are credited. 


\section{References}

1. Jemal A, Bray F, Center MM, Ferlay J, Ward E, Forman D (2011) Global cancer statistics. CA Cancer J Clin 61:69-90

2. Heintz A, Odicino F, Maisonneuve P, Quinn MA, Benedet JL, Creasman WT et al (2006) Carcinoma of the ovary. FIGO 26th annual report on the results of treatment in gynecological cancer. Int J Gynaecol Obstet 95(Suppl 1):S161-S192

3. Bristow RE, Tomacruz RS, Armstrong DK, Trimble EL, Montz FJ (2002) Survival effect of maximal cytoreductive surgery for advanced ovarian carcinoma during the platinum era: a metaanalysis. J Clin Oncol 20:1248-1259

4. Markman M, Markman J, Webster K, Zanotti K, Kulp B, Peterson $\mathrm{G}$ et al (2004) Duration of response to second-line, platinumbased chemotherapy for ovarian cancer: implications for patient management and clinical trial design. J Clin Oncol 22:3120-3125

5. Buda A, Floriani I, Rossi R, Colombo N, Torri V, Conte PF et al (2004) Randomized controlled trial comparing single agent paclitaxel vs epidoxorubicin plus paclitaxel in patients with advanced ovarian cancer in early progression after platinum-based chemotherapy: an Italian collaborative study from the Mario Negri Institute, Milan, G.O.N.O. (Gruppo Oncologico Nord Ovest) group and I.O.R. (Istituto Oncologico Romagnolo) group. Br J Cancer 90:2112-2117

6. Sehouli J, Stengel D, Oskay-Oezcelik G, Zeimet AG, Sommer H, Klare P et al (2008) Nonplatinum topotecan combinations versus topotecan alone for recurrent ovarian cancer: results of a phase III study of the north-eastern German society of gynecological oncology ovarian cancer study group. J Clin Oncol 26:3176-3182

7. Bookman MA, Malmstrom H, Bolis G, Gordon A, Lissoni A, Krebs JB et al (1998) Topotecan for the treatment of advanced epithelial ovarian cancer: an open-label phase II study in patients treated after prior chemotherapy that contained cisplatin or carboplatin and paclitaxel. J Clin Oncol 16:3345-3352

8. Gordon AN, Granai CO, Rose PG, Hainsworth J, Lopez A, Weissman C et al (2000) Phase II study of liposomal doxorubicin in platinum- and paclitaxel-refractory epithelial ovarian cancer. $\mathrm{J}$ Clin Oncol 18:3093-3100

9. Markman M, Webster K, Zanotti K, Kulp B, Peterson G, Belinson J (2003) Phase II trial of single-agent gemcitabine in platinumpaclitaxel refractory ovarian cancer. Gynecol Oncol 90:593-596

10. Sorensen P, Hoyer M, Jakobsen A, Malmström H, Havsteen H, Bertelsen K (2001) Phase II study of vinorelbine in the treatment of platinum-resistant ovarian carcinoma. Gynecol Oncol 81:58-62

11. Brown MR, Blanchette JO, Kohn EC (2000) Angiogenesis in ovarian cancer. Baillieres Best Pract Res Clin Obstet Gynaecol 14:901-918

12. Shimogai R, Kigawa J, Itamochi H, Iba T, Kanamori Y, Oishi T et al (2008) Expression of hypoxia-inducible factor 1alpha gene affects the outcome in patients with ovarian cancer. Int J Gynecol Cancer 18:499-505

13. Klasa-Mazurkiewicz D, Jarzab M, Milczek T, Lipińska B, Emerich J (2011) Clinical significance of VEGFR-2 and VEGFR-3 expression in ovarian cancer patients. Pol J Pathol 62:31-40

14. Ellis LM, Hicklin DJ (2008) VEGF-targeted therapy: mechanisms of anti-tumour activity. Nat Rev Cancer 8:579-591

15. Jain RK (2005) Normalization of tumor vasculature: an emerging concept in antiangiogenic therapy. Science 307:58-62
16. Burger RA, Sill MW, Monk BJ, Greer BE, Sorosky JI (2007) Phase II trial of bevacizumab in persistent or recurrent epithelial ovarian cancer or primary peritoneal cancer: a Gynecologic Oncology group study. J Clin Oncol 25:5165-5171

17. Cannistra SA, Matulonis UA, Penson RT, Hambleton J, Dupont J, Mackey H et al (2007) Phase II study of bevacizumab in patients with platinum-resistant ovarian cancer or peritoneal serous cancer. J Clin Oncol 25:5180-5186

18. McGonigle KF, Muntz HG, Vuky J, Paley PJ, Veljovich DS, Greer BE et al (2011) Combined weekly topotecan and biweekly bevacizumab in women with platinum-resistant ovarian, peritoneal, or fallopian tube cancer: results of a phase 2 study. Cancer 117:3731-3740

19. Tillmanns TD, Lowe MP, Walker MS, Stepanski EJ, Schwartzberg LS (2013) Phase II clinical trial of bevacizumab with albumin-bound paclitaxel in patients with recurrent, platinum-resistant primary epithelial ovarian or primary peritoneal carcinoma. Gynecol Oncol 128:221-228

20. Verschraegen CF, Czok S, Muller CY, Boyd L, Lee SJ, Rutledge T et al (2012) Phase II study of bevacizumab with liposomal doxorubicin for patients with platinum- and taxane-resistant ovarian cancer. Ann Oncol 23:3104-3110

21. Elit L, Hirte H (2013) Palliative systemic therapy for women with recurrent epithelial ovarian cancer: current options. Onco Targets Ther 6:107-118

22. Pujade-Lauraine E, Hilpert F, Weber B, Reuss A, Poveda A, Kristensen $\mathrm{G}$ et al (2014) Bevacizumab combined with chemotherapy for platinum-resistant recurrent ovarian cancer: the AURELIA open-label randomized phase III trial. J Clin Oncol 32:1302-1308

23. Emile G, Chauvenet L, Tigaud JM, Chidiac J, Pujade Lauraine E, Alexandre J (2013) A clinical experience of single agent bevacizumab in relapsing ovarian cancer. Gynecol Oncol 129:459-462

24. Perren TJ, Swart AM, Pfisterer J, Ledermann JA, Pujade-Lauraine E, Kristensen $\mathrm{G}$ et al (2011) A phase 3 trial of bevacizumab in ovarian cancer. N Engl J Med 365:2484-2496

25. Matsumoto K, Katsumata N, Yamanaka Y, Yonemori K, Kohno T, Shimizu C et al (2006) The safety and efficacy of the weekly dosing of irinotecan for platinum- and taxanes-resistant epithelial ovarian cancer. Gynecol Oncol 100:412-416

26. Yonemori K, Katsumata N, Yamamoto N, Kasamatsu T, Yamada T, Tsunematsu R et al (2005) A phase I study and pharmacologic evaluation of irinotecan and carboplatin for patients with advanced ovarian carcinoma who previously received platinumcontaining chemotherapy. Cancer 104:1204-1212

27. Therasse P, Arbuck SG, Eisenhauer EA, Wanders J, Kaplan RS, Rubinstein L et al (2000) New guidelines to evaluate the response to treatment in solid tumors: european organization for research and treatment of cancer, National Cancer Institute of the United States, National Cancer Institute of Canada. J Natl Cancer Inst 92:205-216

28. Common Terminology Criteria for Adverse Events (CTCAE) Version 4.0. The CTCAE register. http://evs.nci.nih.gov/ftp1/ CTCAE/CTCAE_4.03_2010-06-14_QuickReference_5x7.pdf

29. Burger RA (2011) Overview of anti-angiogenic agents in development for ovarian cancer. Gynecol Oncol 121:230-238

30. Bodurka DC, Levenback C, Wolf JK, Gano J, Wharton JT, Kavanagh JJ et al (2003) Phase II trial of irinotecan in patients with metastatic epithelial ovarian or peritoneal cancer. J Clin Oncol 21:291-297 